\title{
Brain microvascular endothelial cell dysfunction in an isogenic juvenile iPSC model of Huntington's disease
}

\author{
Raleigh Linville \\ Johns Hopkins University \\ Renée Nerenberg \\ Johns Hopkins University \\ Gabrielle Grifno \\ Johns Hopkins University \\ Diego Arevalo \\ Johns Hopkins University \\ Zhaobin Guo \\ Johns Hopkins University \\ Peter Searson ( $\nabla$ searson@jhu.edu ) \\ Johns Hopkins University https://orcid.org/0000-0002-5417-0828
}

\section{Research}

Keywords: blood-brain barrier, Huntington's disease, brain microvascular endothelial cells, neurodegenerative disease, induced pluripotent stem cells

Posted Date: September 22nd, 2021

DOl: https://doi.org/10.21203/rs.3.rs-903286/v1

License: (c) (i) This work is licensed under a Creative Commons Attribution 4.0 International License. Read Full License 


\section{Abstract}

Huntington's disease (HD) is an inherited neurodegenerative disease caused by expansion of cytosineadenine-guanine (CAG) repeats in the huntingtin gene, which leads to neuronal loss and decline in cognitive and motor function. Increasing evidence suggests that blood-brain barrier (BBB) dysfunction may contribute to progression of the disease. Studies in animal models, in vitro models, and post-mortem tissue suggest that disease progression is associated with increased microvascular density, altered cerebral blood flow, and loss of paracellular and transcellular barrier function. Here we report on changes in BBB phenotype due to expansion of CAG repeats using an isogenic pair of induced pluripotent stem cells (iPSCs) differentiated into brain microvascular endothelial-like cells (iBMECs). We show that CAG expansion alters the trajectory of iBMEC differentiation, producing cells with $\sim 2$-fold lower purity of adherent endothelial cells. CAG expansion is associated with lower transendothelial electrical resistance, lower tight junction protein expression, and unique gene expression profiles, but no significant changes in paracellular permeability. In addition, CAG expansion results in unique responses to pathological and therapeutic perturbations including angiogenic factors, oxidative stress, and osmotic stress. In a tissueengineered BBB model, iBMECs show subtle changes in phenotype, including differences in cell turnover and immune cell adhesion. Our results further support that CAG expansion in BMECs may alter BBB phenotype during HD.

\section{Introduction}

Huntington's disease (HD) is an inherited autosomal dominant neurodegenerative disease that affects 1 in 10,000 Americans and causes cognitive deficits and loss of motor function that are ultimately fatal [1]. $\mathrm{HD}$ is caused by the expansion of cytosine-adenine-guanine (CAG) repeats in the huntingtin gene (HTT), which leads to the production of mutant huntingtin protein (MHTT). Loss of normal HTT and gain of $\mathrm{mHTT}$ leads to neuronal loss, accompanied by cognitive and motor dysfunction [2].

The human blood-brain barrier (BBB) is comprised of brain microvascular endothelial cells (BMECs), along with supporting cells, that maintain neuronal homeostasis. There is accumulating evidence from in vitro and in vivo studies that HD is associated with dysfunction of the BBB [3-6]. Cerebrovascular changes are observed in animal models and post-mortem human tissue including increased microvascular density $[3,7,8]$, BBB breakdown $[3,4]$, and altered cerebral hemodynamics [8-10]. Additionally, in vitro studies using human induced pluripotent stem cell (iPSC)-derived BMEC-like cells (iBMECs) have found that CAG expansion elevates angiogenic potential, reduces paracellular barrier strength, and changes transcellular efflux $[5,6]$. These cerebrovascular changes may contribute to the early pathogenesis of HD and represent a possible therapeutic target. However, many aspects of BMEC phenotype remain to be explored and, to date, isogenic controls have not been utilized for these studies. To expand the understanding of changes in BMEC phenotype that may contribute to HD pathogenesis, we build on previous reports by: (1) utilizing an isogenic pair of iPSCs to directly determine effect of CAG mutation on differentiation trajectory and resulting iBMEC phenotype, (2) utilizing two-dimensional (2D) and three-dimensional (3D) BBB models to confirm results in the presence of physiological cues (i.e. 
shear stress), and (3) by validating our results across multiple differentiations and protocol variables, to overcome uncertainties associated with lab-to-lab differences.

We differentiated iBMECs from a juvenile HD patient with 180 CAG repeats and an isogenic control in which the CAG expansion was corrected using CRISPR/Cas9 gene editing [6]. Recent work suggests a neurodevelopmental component to HD progression [11], and juvenile-derived CRISPR/Cas9-corrected iPSCs were previously used to show reversal of phenotypic abnormalities in iPSC-derived neurons [12]. This approach is distinct from existing work utilizing adult HD iPSCs which harbor aged-induced epigenetic changes [5]. We found that CAG expansion reduced transendothelial electrical resistance (TEER) of HD iBMECs ( 3-fold), corresponding with mislocalization of tight junction proteins but no difference in paracellular permeability to small molecular weight compounds. Additionally, by screening phenotype across optimized differentiation variables (initial seeding density, transwell seeding density, and media composition), we confirmed that CAG expansion was associated with slightly reduced TEER and further validated using non-isogenic HD iPSCs. Critically, other aspects of BBB phenotype were altered by CAG expansion including decreased efflux activity, increased sensitivity to angiogenic, oxidative, and osmotic factors, dysregulated cell turnover, and increase immune cell adhesion.

\section{Materials And Methods}

\section{Cell culture}

Four induced pluripotent stem cell (iPSC) sources were used in this work: juvenile-onset HD iPSCs with 180 CAG repeats (HD180) [12], isogenic CRISPR-corrected controls of HD180 with 18 CAG repeats (HDcorrected) [12], non-isogenic adult onset HD iPSCs with 50 CAG repeats (HD50) (from NINDS cell repository \#NN0003930), and non-isogenic control iPSCs with 21 CAG repeats (HD21) (from Allen Cell Institute \#AICS-0023). The two isogenic cells were provided by the Pouladi Lab at the National University of Singapore. Details of each cell line are summarized in Table S1. Cell culture was performed at $37^{\circ} \mathrm{C}$ and $5 \% \mathrm{CO}_{2}$. iBMECs were differentiated from iPSCs using protocols developed in the Searson Group [13, 14]. Briefly, iPSC colonies were formed by seeding iPSCs singularized with Accutase (Invitrogen) at 10,000 cells $\mathrm{cm}^{-2}$ (additional seeding densities outlined below) and growing in mTeSR1 or TeSR-E8 (Stem Cell Technologies) for three days on six-well plates. Colonies were then treated with UM/F- media (DMEM/F12, 20\% knockout serum replacement, 1\% non-essential amino acids, 0.5\% GlutaMAX, and $0.836 \mu \mathrm{M}$ beta-mercaptoethanol) for six days, and endothelial media (human endothelial cell serum-free medium, $1 \%$ human platelet poor derived serum, $2 \mathrm{ng} \mathrm{mL}^{-1} \mathrm{bFGF}$, and $10 \mathrm{mM}$ all-trans retinoic acid) for two days. iPSC media was switched daily using a media volume of $2 \mathrm{~mL}$; UM/F- and endothelial media were switched daily using a media volume of $1 \mathrm{~mL}$. At various stages of the differentiation, viable cells were manually counted on a hemacytometer based on Trypan blue (Corning) exclusion. At the end of the differentiation, cells were singularized using a 30-minute treatment with Accutase. Adherent cells were isolated by sub-culture on a plate coated overnight with $50 \mu \mathrm{g} \mathrm{mL}^{-1}$ human placental collagen IV (Sigma) and $25 \mu \mathrm{g} \mathrm{mL}^{-1}$ fibronectin from human plasma (Sigma). This process was conducted for one hour in 
endothelial media supplemented with $1 \%$ penicillin-streptomycin (ThermoFisher Scientific) and $10 \mu \mathrm{M}$ ROCK inhibitor Y27632 (ATCC). Following subculture, the monolayer of adherent cells was washed with phosphate-buffered saline (PBS; ThermoFisher) and then singularized using a 10-minute treatment with Accutase. Viable cells were seeded onto collagen IV and fibronectin-coated surfaces at $0.33 \times 10^{6}$ cells $\mathrm{cm}^{-2}$. For the first $24 \mathrm{~h}$ of culture, the media matched that of subculture but was then replaced with basal media (human endothelial cell serum-free medium, $1 \%$ human platelet poor derived serum, and $1 \%$ penicillin-streptomycin). HD180 and HD-corrected iPSCs were confirmed to be isogenic using a PowerPlex 18D kit (Promega). A PCR based MycoDtect kit (Greiner Bio-One) was used to confirm absence of mycoplasma.

\section{Differentiation variables}

Beyond the differentiation scheme presented above, differentiation variables were adjusted to determine effects on differentiation outcomes and iBMEC phenotype. These variables included: (1) initial iPSC seeding density, (2) transwell seeding density, (3) removal of the sub-culture step before seeding for experiments, (4) media volume used during differentiation, and (5) use of a serum-free medium alternative during differentiation and transwell culture. To test the effect of initial seeding density on differentiation outcomes, hiPSCs were passed using the technique described previously, but seeded at densities of $5,10,20,30,40 \times 10^{3}$ cells $\mathrm{cm}^{-2}$ in parallel on Matrigel-coated plates. To test the effect of transwell seeding density on the barrier function of iBMECs, the cells were harvested using the technique described previously, and seeded on transwells at the densities of 0.33 and $1 \times 10^{6} \mathrm{cells} \mathrm{cm}^{-2}$ (3-fold difference in density) without the use of a sub-culture purification step. To determine the effect of the media volume used during differentiation, cells were grown in either 1 or $2 \mathrm{~mL}$ of UM/F- and RA media throughout the duration of the differentiation. To determine the effect of performing a serum-free differentiation, the $1 \%$ human platelet poor derived serum in endothelial media used in the final two days on the differentiation and during transwell culture was replaced with 1\% B-27 Supplement (ThermoFisher), as previously demonstrated [15].

\section{Quantitative polymerase chain reaction (qPCR)}

Quantitative PCR (qPCR) was conducted on an Applied Biosystems StepOnePlus Real-Time PCR system using TaqMan ${ }^{\circledR}$ probes. Confluent monolayers of iBMECs two days after seeding on 6-well plates were washed with PBS and lysed using RLT buffer (Qiagen RNeasy Mini Kit). Fold changes between HD180 and $\mathrm{HD}$-corrected were calculated using the comparative $\mathrm{CT}$ method $(\triangle \triangle \mathrm{Ct})$ normalizing to ACTB expression (housekeeping gene). The following genes and primers were used: CLDN5 (\#Hs00533949), OCLN (\#Hs00170162), SLC2A1 (\#Hs00892681), ABCB1 (\#Hs00184500), PECAM1 (\#Hs00169777), CDH5 (\#Hs00901463), ACTB (\#Hs01060665).

\section{Immunocytochemistry}

iBMECs were seeded at 250,000 cells $\mathrm{cm}^{-2}$ on borosilicate cover glass slides and cultured for two days using media outlined above. iBMECs were then washed with PBS, fixed with methanol for $15 \mathrm{~min}$, and blocked with $10 \%$ goat serum (Cell Signaling Technology) or 10\% donkey serum (Millipore Sigma) 
supplemented with $0.3 \%$ Triton X-100 (Millipore Sigma) in PBS for 30 min. Primary antibodies are summarized in Table S2. Cells were treated with Alexa Flour-647 and Alexa Flour-488 conjugated secondary antibodies (Life Technologies) diluted 1:200 in blocking buffer for $45 \mathrm{~min}$ at room temperature. To localize nuclei, cells were treated with $1 \mathrm{\mu g} \mathrm{mL}^{-1} \mathrm{DAPI}$ (ThermoFisher). Images were acquired at 40x magnification using a swept field confocal microscope (Bruker).

\section{RNA sequencing}

Two biological replicates were analyzed of: HD180 iPSCs, HD180 iBMECs, HD-corrected iPSCs, and HDcorrected iBMECs. iPSCs were harvested prior to differentiation in UM/F- media, while iBMECs were harvested as confluent monolayers two days following subculture on collagen IV and fibronectin-coated tissue-culture plates. To harvest total RNA, cells were lysed using RLT buffer (Qiagen) supplemented with $\beta$-mercaptoethanol (Sigma) and then RNA isolated using a RNeasy Mini Kit (Qiagen) with DNase I digestion (Qiagen). All sequenced samples had RNA integrity numbers above 9.7, as measured by an Agilent 2100 bioanalyzer. Total RNA was subjected to oligo (dT) capture and enrichment, and the resulting mRNA fraction was used to construct CDNA libraries. Approximately 20 million paired end 150 bp reads were collected per sample using Illumina NovoSeq (performed by Novogene). Alignment and quantification to reference genome (GRCh38) was performed using Rsubread (Version 2.0.1) [16]. Normalization (rlog transformed), visualization, and differential analysis was performed using DESeq2 (v1.28.1) [17]. Differentially expressed genes (DEGs) were determined using the Wald test with BenjaminiHochberg correction (adjusted $p$ values $<0.05$ was considered statistically significant). Pathway enrichment analysis (Hallmark gene sets) was conducted on DEGs using Enrichr, with built in statistical analysis used at a adjusted p-value cutoff of 0.05 [18]. Raw data are deposited at NCBI.

\section{Barrier function measurements}

Transendothelial electrical resistance (TEER; $\Omega \mathrm{cm}^{2}$ ) was recorded using an EndOhm (World Precision Instruments) as previously reported [14]. Measurements were performed on $6.5 \mathrm{~mm}$ Transwells with a 0.4 $\mu \mathrm{m}$ pore polyester membrane insert (Corning). TEER values were corrected for the resistance of the transwell insert without cells. iBMECs were seeded at a density of $0.33-1.00 \times 10^{6} \mathrm{~cm}^{-2}$ onto transwells in endothelial media as previously described. After 24 hours, medium was switched to basal medium and daily recordings were collected for ten days.

At day two (and sometimes day ten), the permeability of $200 \mu \mathrm{M}$ Lucifer yellow (ThermoFisher), $2 \mu \mathrm{M} 10$ kDa dextran (ThermoFisher), $10 \mu \mathrm{M}$ rhodamine 123 (ThermoFisher), and $25 \mathrm{mM}$ d-glucose (Sigma) across BMEC monolayers was measured using previously reported protocols [14]. The following excitation and emission settings were utilized on a Synergy ${ }^{\mathrm{Tm}} \mathrm{H} 4$ microplate reader (Biotek): Lucifer yellow $(428 \mathrm{~nm} / 545 \mathrm{~nm}), 10 \mathrm{kDa}$ dextran $(647 \mathrm{~nm} / 667 \mathrm{~nm})$, rhodamine 123 (495 nm / $525 \mathrm{~nm})$. Glucose transport was quantified using a glucose colorimetric detection kit (ThermoFisher Scientific), utilizing absorbance measurements at $560 \mathrm{~nm}$. Concentrations of all compounds were determined from calibration curves based on serial dilution of each compound spanning four orders of magnitude. The apparent permeability of each compound was calculated as $P=(d C / d t)(V)(1 / A)\left(1 / C_{0}\right)$, where $d C / d t$ is the 
slope of cumulative concentration, $V$ is the volume of the receiving compartment (i.e. basolateral or apical chamber), $A$ is the area of the monolayer, and $C_{0}$ is the dosed concentration of solute [19]. For rhodamine 123, efflux ratios were calculated as the ratio of basolateral-to-apical and apical-to-basolateral permeability normalized to $10 \mathrm{kDa}$ dextran (a non-efflux substrate). Biological replicates of permeability measurements were averaged across at least two transwells (technical replicates).

\section{Responsiveness to chemical perturbation}

A bead angiogenesis assay was conducted as previously reported [20]. Briefly, $150 \mu \mathrm{m}$ diameter Cytodex ${ }^{\mathrm{TM}}$ 3 microcarrier beads (GE Healthcare) were coated with collagen IV and fibronectin and then seeded with singularized iBMECs in endothelial media supplemented with $1 \%$ penicillin-streptomycin and $10 \mu \mathrm{M}$ ROCK inhibitor. After 80 min, with gentle agitation every $20 \mathrm{~min}$, the beads were washed to remove nonadherent cells and then cultured on a shaker at $100 \mathrm{rpm}$ for $24 \mathrm{~h}$. Next, beads were embedded within 6 $\mathrm{mg} \mathrm{mL}^{-1}$ neutralized rat tail type I collagen (Corning) and treated with basal media with and without $50 \mathrm{ng}$ $\mathrm{mL}^{-1}$ recombinant human VEGF-165 (VEGF; Biolegend). After three days in culture, the sprout density (\# bead $^{-1}$ ) was manually counted from phase contrast images across at least 8 beads (technical replicates) for each condition.

For oxidative and osmotic stress experiments, iBMECs were seeded onto transwells as previously described and exposed to hydrogen peroxide $\left(\mathrm{H}_{2} \mathrm{O}_{2}\right)$ or mannitol (for $10 \mathrm{mins}$ ) after $48 \mathrm{~h}$. To avoid the need for a media switch, $5 \mu \mathrm{L}$ of concentrated $\mathrm{H}_{2} \mathrm{O}_{2}$ freshly prepared in sterile water was added to the apical chamber of transwells to achieve final concentrations of $0.2-1 \mathrm{mM}$. TEER was recorded daily after exposure. As mannitol only induces BBB opening near its concentration limit, the medium was changed to basal medium with $1.4 \mathrm{M}$ mannitol for $10 \mathrm{~min}$, and then switched back to basal medium. TEER was recorded immediately before treatment, immediately after treatment, one hour later and one day later. To visualize reactive oxygen species and actin cytoskeleton, some transwells were treated with $50 \mu \mathrm{M}$ CellROX® Green Reagent (Invitrogen), AlexaFluor647 phalloidin (Invitrogen), and DAPI solution (Thermo Scientific) for 30 min at $37^{\circ} \mathrm{C}$ after one day of exposure to $0.6 \mathrm{mM} \mathrm{H}_{2} \mathrm{O}_{2}$.

\section{Tissue-engineered BBB model}

Tissue-engineered BBB microvessels were fabricated as previously reported [21]. iBMECs were subcultured for one hour and then detached using Accutase before seeding into $150 \mu \mathrm{m}$ diameter channels patterned in $7 \mathrm{mg} \mathrm{mL}^{-1}$ type I collagen. Prior to seeding, the collagen matrix was cross-linked with $20 \mathrm{mM}$ genipin to increase stiffness and then the channel surface was coated in collagen IV and fibronectin to promote cell adhesion. Cells were seeded into microvessels and cultured for 30 min under no flow to facilitate adhesion, then microvessels were perfused at $\sim 1$ dyne $\mathrm{cm}^{-2}$ shear stress for the remainder of experimentation. After formation of confluent monolayers, microvessels were perfused with $200 \mu \mathrm{M}$ Lucifer yellow for one hour, with images collected every two minutes. From the plot of fluorescence over time, permeability was calculated as previously reported [22]. The turnover of iBMECs in microvessels was calculated from phase contrast images acquired simultaneously with fluorescence images. Cell loss and cell proliferation events were manually counted on the top plane of the microvessel as previously 
reported [21]. From counts of cell loss and proliferation events, values were normalized to total number of cells in the imaging plane and to time, with final units of $\% \mathrm{~h}^{-1}$. Net microvessel turnover was calculated as the difference in rate between proliferation and loss $\left(\% \mathrm{~h}^{-1}\right)$.

THP-1 (ATCC $®$ TIB-202 ${ }^{\text {TM }}$ ) is a human leukemia monocytic cell line [23]. THP-1s were grown in suspension with RPMI-1640 Medium (Sigma) supplemented with 10\% fetal bovine serum (Sigma) and $1 \%$ penicillin-streptomycin. Before use, cells were labeled with $5 \mu \mathrm{M}$ Calcein AM (ThermoFisher) for 15 min, and then resuspended at $1 \times 10^{6}$ cells $\mathrm{mL}^{-1}$ in basal media. Microvessels were perfused with THP-1s under low shear stress $\left(\sim 0.2\right.$ dyne $\left.\mathrm{cm}^{-2}\right)$ for $10 \mathrm{~min}$, and then washed out using higher shear stress $(\sim 2$ dyne $\mathrm{cm}^{-2}$ ). Adherent immune cells were manually counted along the length of microvessels using fluorescence microscopy.

\section{Statistical analysis}

All statistical analysis was performed using Prism ver. 8 (GraphPad). Metrics are presented as mean \pm SEM (standard error of the mean). A student's unpaired t-test (two-tailed with unequal variance) was used for comparison of two groups, and an analysis of variance (ANOVA) for comparison of three or more groups. Number of biological replicates are reported in figure legends. P-values were multiplicity adjusted using a Tukey test. Differences were considered statistically significant for $p<0.05$, with thresholds of * $p$ $<0.05$, ** $p<0.01$, and $* \star * p<0.001$.

\section{Results}

\section{hiPSCs with elevated CAG repeats display a unique BMEC differentiation trajectory}

Brain microvascular endothelial-like cells (iBMECs) were differentiated from an isogenic pair of iPSCs with 180 (HD180) and 18 (HD-corrected) CAG repeats in the HTT gene. Differentiation was conducted by sequential treatment with mTeSR1 for three days, UM/F- for six days, and endothelial media (RA) for two days, as previously reported $[13,14]$ (Fig. 1A). Next, cells are purified by subculture on collagen IV and fibronectin-coated plates and detached for seeding onto glass, transwells, or tissue-engineered microvessels. The differentiation of the HD180 iPSCs was visibly unique compared to isogenic HDcorrected iPSCs and other control iPSCs; while HD180 iPSC colonies appeared similar, treatment with UM/F- resulted in limited neural tracts, which is a hallmark of iBMEC differentiation (dotted red line; Fig. 1B). Additionally, following sub-culture to selectively purify iBMECs, differentiation of HD180 iPSCs produced significantly fewer adherent cells compared to the isogenic control (Fig. 1C). Following the three days of growth in mTeSR1, we observed a higher density of HD180 iPSCs $(p=0.005)$. In contrast, following differentiation and sub-culture we observed a higher density of HD-corrected cells $(p<0.001$ for both comparisons) (Fig. 1D). Additionally, the overall purity of the differentiation (defined as the number of adherent cells following sub-culture divided by the number of differentiated cells) was lower for the HD180 iPSC differentiation ( $p=0.038$ ) (Fig. 1E). These results suggest a unique differentiation trajectory 
for iPSCs with expanded elevated CAG repeats. However, adherent cells from both iPSC sources stained uniformly for platelet endothelial cell adhesion molecule (CD31) and glucose-transporter 1 (GLUT-1) (Fig. $1 F)$. Additionally, across both differentiations there was a loss in gene expression of pluripotency markers (POU5F1, SOX2, MYC) and gain in gene expression of VE-cadherin (CDH5), GLUT-1 (SLC2A1), and retinoic acid receptor alpha (RARA) (Fig. 1G). CD31, VE-cadherin, and GLUT-1 expression have been utilized to assess differentiation efficiency [5,24], while RARA upregulation was shown to induce barrier function following iBMEC differentiation [25]. Together these results suggest that independent of CAG expansion, the differentiation produced brain endothelial-like cells.

\section{CAG expansion alters barrier function}

We characterized iBMEC monolayers via immunocytochemistry and QPCR of various BBB markers, as well as functional measurements of transendothelial electrical resistance (TEER) and permeability (Fig. 2). HD180 iBMECs displayed decreased staining of the junctional proteins occludin, zonula occludens-1 (ZO1), and VE-cadherin, while claudin-5 staining was similar, and P-gp staining was increased compared to HD-corrected iBMECs (Fig. 2A). However, localization of tight junction proteins in HD180 iBMECs was unchanged, despite the decreased staining (Fig. S1). These observations were generally matched by qPCR, where key BBB genes were expressed at lower levels in HD180 iBMECs (Fig. 2B). While claudin-5 protein localization appeared similar, gene expression was much lower in the HD180 iBMECs. P-gp protein expression was higher in HD180s, while gene expression was slightly lower. The HD-corrected cells exhibited a typical TEER time course [13], with peak values of $\sim 3,000 \Omega \mathrm{cm}^{2}$ on day two followed by a gradual decrease. The HD180 cells exhibited a unique trajectory, with $\sim$ 3-fold lower TEER on day 2 ( $p<$ 0.001). Both HD180s and HD-corrected iBMECs exhibited TEER values $>1,000 \Omega \mathrm{cm}^{2}$ from days $6-10$ (Fig. 2C). The average TEER over ten days was $\sim 1,024 \Omega \mathrm{cm}^{2}$ and $\sim 2,067 \Omega \mathrm{cm}^{2}$ for HD180s and HDcorrected iBMECs, respectively $(p<0.001)$ (Fig. 2D). Despite differences in TEER, the permeability of Lucifer yellow (444 Da) was independent of CAG expansion at both day two $(p=0.171)$ and day ten $(p=$ 0.606) (Fig. 2E), indicating preserved paracellular permeability despite CAG expansion. Permeability measurements with $10 \mathrm{kDa}$ dextran matched observations with Lucifer yellow (Fig. 2F). In transwells, Lucifer yellow permeability was $\sim 2 \times 10^{-6} \mathrm{~cm} \mathrm{~s}^{-1}$ on day two and $\sim 4 \times 10^{-6} \mathrm{~cm} \mathrm{~s}^{-1}$ on day ten, while the permeability of $10 \mathrm{kDa}$ dextran was $\sim 6 \times 10^{-8} \mathrm{~cm} \mathrm{~s}^{-1}$ on days 2 and 10 . These values match previous measurements of iBMEC permeability across other iPSC sources [14]. We also measured the efflux ratio (ratio of apical-to-basolateral to basolateral-to-apical permeability) of rhodamine 123 (R123), a substrate of the P-gp efflux pump. HD180 iBMECs exhibited lower efflux ratios of R123 than HD-corrected cells ( $p=$ 0.005 ) (Fig. 2G). Both HD-corrected and HD180 iBMECs displayed similar permeability to glucose ( $p=$ 0.970) (Fig. 2H).

All recordings in panel C-G represent averages across $n=2-6$ technical replicates (individual transwells) for each biological replicate.

\section{Changes in barrier function are independent of differentiation variables}


iBMEC differentiation is sensitive to variables including reagent source, seeding density, and serum lot $[15,26,27]$. Therefore, we sought to determine whether differentiation variables would alter differences in phenotype between HD-corrected and HD180 iBMECs. We tested the effects of iPSC seeding density, transwell seeding density, media volume, and use of serum free alternatives.

After seeding iPSCs at $5,000,10,000,20,000,30,000$, and 40,000 cells $\mathrm{cm}^{-2}$ we counted viable cells postmTeSR1, post-RA, and post-subculture (Fig. 1A). After mTeSR1 (before starting the iBMEC differentiation), HD180 and HD-corrected cells showed similar iPSC cell counts at high initial seeding densities $(>10,000$ cells $\left.\mathrm{cm}^{-2}\right)$, while HD180 iPSCs were more populous at lower seeding densities $\left(\leq 10,000\right.$ cells $\left.\mathrm{cm}^{-2}\right)(p<$ 0.01 for both comparisons) (Fig. S2A). Post-differentiation, the HD180 line produced significantly fewer cells before (post-RA) and after subculture, regardless of the initial seeding density $(p<0.01$ for all comparisons) (Fig. S2B-C). Subculture purity was maximized at lower initial seeding densities (5,000 and 10,000 cells $\mathrm{cm}^{-2}$ ), although the purity (fraction of adherent cells) for HD180 cells was significantly lower $(p<0.05)$ (Fig. S2D).

Across all initial seeding densities and both cell sources, we seeded transwells directly with $0.33 \times 10^{6}$ and $1.0 \times 10^{6}$ cells $\mathrm{cm}^{-2}$. In addition, we performed a subculture purification prior to seeding transwells at $0.33 \times 10^{6}$ cells $\mathrm{cm}^{-2}$. Statistically significant differences in TEER were observed across all different seeding approaches at the initial seeding density of 10,000 cells $\mathrm{cm}^{-2}(p<0.05$ for all comparisons) (Fig. S3).

We also examined the effect of increased media volume during the differentiation and the use of the serum replacement B-27 (Fig. S4). We found that performing the differentiation in $2 \mathrm{~mL}$ of media decreased the average TEER significantly for the HD180 $(p=0.013)$ but not significantly for HD-corrected cells $(p=0.247)$. Interestingly, the use of a serum-free differentiation decreased average TEER, contrary to previous reports [15]; the average TEER was significantly lower in the HD180 cells $(p=0.021)$ but not significantly lower in the HD-corrected cells $(p=0.352)$.

\section{Non-isogenic control and HD iPSCs corroborate findings}

In healthy individuals the average HTT gene CAG repeat length is 20 [28]. To compare changes in iBMEC phenotype across a broader range of CAG repeat lengths, we tested barrier function of two additional non-isogenic iPSCs: (1) an adult-onset HD iPSC line with 50 CAG repeats (HD50), and (2) a control iPSC line with 21 CAG repeats (HD21) (Table S1). Differentiations were conducted matching the optimized protocol for HD180 and HD-corrected iPSCs (i.e. initial seeding density of 10,000 cells cm${ }^{-2}$, subculturing to purify cells, and seeding on transwells at $0.33 \times 10^{6}$ cells $\mathrm{cm}^{-2}$ ). Following differentiation, the fraction of adherent iBMECs was CAG-length dependent across all iPSCs (Fig. S5A). Similarly, TEER values were higher for the lower CAG lengths (18 and 21), although not statistically significant (Fig. S5B). These results suggest that moderate reductions in TEER are not unique to the specific isogenic iPSC line used in this study. 


\section{CAG expansion uniquely alters gene expression of HD iBMECs}

Huntington's disease results in widespread transcriptional dysregulation in the brain [29-31]. Bulk RNA sequencing was utilized to compare global gene expression profiles between HD180 and HD-corrected iPSCs, and the corresponding HD180 and HD-corrected iBMECs (Fig. 3). Principle component analysis (PCA) showed that distinct gene expression profiles emerged following iBMEC differentiation (Fig. 3A). We identified 177 upregulated and 95 downregulated genes between HD180 and HD-corrected iPSCs; however, gene set enrichment analysis (GSEA) using hallmark gene sets from the Molecular Signatures Database (MSigDB) found only a single gene set significantly enriched (Epithelial Mesenchymal Transition; enriched in HD180 iPSCs) (Fig. 3B). In contrast, we identified 1,397 upregulated and 982 downregulated genes between HD180 and HD-corrected iBMECs, corresponding to 18 enriched and 10 depleted hallmark gene sets in HD180 iBMECs (Fig. 3B). Less than 5\% of up and downregulated genes were shared between iPSCs and iBMECs, suggesting a distinct impact of CAG expansion on the two cell types (Fig. 3C). Enriched hallmark gene sets for HD180 iBMECs included those involved in cell growth and division (G2-M checkpoint, mitotic spindle), hypoxia, immune response (IL-2/STAT5 signaling, interferon gamma response), and angiogenesis, whereas depleted hallmark gene sets included those involved in canonical brain endothelial functions including coagulation, apical junction, wnt-beta catenin signaling, TNF-alpha signaling via NF-kB, and complement (Fig. 3D).

\section{Responsiveness to oxidative, angiogenic, and osmotic stimuli}

Since the cellular processes associated with enriched gene sets are also associated with HD disease progression, we sought to determine how HD iBMECs responded to related pathological perturbations. Here, we assessed the response of HD180 iBMECs to oxidative, angiogenic, and osmotic stress (Fig. 4A). BMECs can be exposed to reactive oxygen species (ROS) or vascular endothelial growth factor (VEGF) generated in the brain parenchyma or present in circulation [8,32]. Increased oxidative stress markers are detected in peripheral blood of HD patients and asymptomatic HD gene carriers [33-35]. Previous studies have found that the iPSC-derived microglia and neurons harboring expanded CAG repeats release elevated levels of ROS and are hypersensitive to exogenous stress [36, 37]. We evaluated the effect of oxidative stress on iBMECs exposed to a range of $\mathrm{H}_{2} \mathrm{O}_{2}$ concentrations $(0.2-1 \mathrm{mM})$ by measuring TEER [38]. $\mathrm{H}_{2} \mathrm{O}_{2}$ can exert concentration-dependent effects on BMEC phenotype, including induction of apoptosis or angiogenesis [20,39]. There was a sharp decrease in TEER values at an $\mathrm{H}_{2} \mathrm{O}_{2}$ concentration greater than $0.6 \mathrm{mM}$ for both HD-corrected and HD180 iBMECs (Fig. 4B). This concentration is within the range where pathological effects are observed following inhalation or ingestion [40]. Following exposure to $0.6 \mathrm{mM} \mathrm{H}_{2} \mathrm{O}_{2}$ for $24 \mathrm{~h}$, HD180 iBMECs showed dramatically reduced TEER compared to vehicle $(\sim 150$

$\left.\Omega \mathrm{cm}^{2}\right)(p=0.008)$ (Fig. 4B). In contrast, the was no statistical difference in TEER between $0.6 \mathrm{mM} \mathrm{H}_{2} \mathrm{O}_{2}$ 
and vehicle for HD-corrected iBMECs ( $p=0.095$ ) (Fig. 4C). Staining of HD180 iBMECs exposed to $0.6 \mathrm{mM}$ $\mathrm{H}_{2} \mathrm{O}_{2}$ revealed gaps in the monolayer, whereas HD-corrected monolayers remained intact (Fig. 4D).

Post-mortem HD tissue is characterized by an increase in angiogenic microvessels [3, 4], while increased astrocytic secretion of VEGF-A is observed in HD mouse models (R6/2) [8]. An increased angiogenic phenotype has also been inferred from an in vitro wound healing assay in HD-iBMEC monolayers [5]. To test angiogenic potential, we performed a bead angiogenesis assay by coating $150 \mu \mathrm{m}$ diameter beads with iBMECs [20], embedding beads within collagen I and Matrigel after formation of a confluent monolayer, and exposing the beads to $50 \mathrm{ng} \mathrm{mL}^{-1}$ vascular endothelial growth factor. HD180 iBMECs displayed elevated VEGFR2 protein expression compared to HD-corrected cells (Fig. 4E). Upon exposure to VEGF, sprout density increased for HD180 cells $(p=0.002)$, but was unchanged for HD-corrected iBMECs $(p=0.726)($ Fig. $4 F, G)$.

While recent therapeutic approaches for HD utilize intrathecal delivery to bypass the BBB, BBB opening (BBBO) represents a possible strategy to increase drug delivery to neurons following intravenous delivery. Osmotic BBBO utilizes intra-arterial (IA) infusion of hyperosmotic agents to transiently disrupt cell-cell junctions thereby enabling delivery of large molecular weight compounds to the brain [41, 42]. We hypothesized that the HD BBB may have unique susceptibility to BBBO. Indeed, recent work found that AD-iBMECs (with PSEN1 mutations) display altered responsiveness to focused ultrasound (FUS), another strategy for transient BBBO [43]. To test sensitivity to osmotic BBBO, we treated iBMEC monolayers with clinical concentrations of the hyperosmotic agent mannitol (1.4 M) for ten minutes. Independent of CAG expansion mannitol treatment reversibly decreased TEER, with recovery to baseline values by one day after exposure (Fig. 4H). However, HD180 iBMECs displayed 4-fold lower TEER immediately following mannitol treatment $(p=0.011)$ and one hour later $(p=0.029)$, suggesting that drug delivery across the HD BBB may be elevated compared to the healthy BBB.

\section{Tissue-engineered HD BBB microvessels}

To study barrier function and endothelial cell turnover in real-time we generated three-dimensional tissueengineered microvessels, as previously reported (Fig. 5A) [21]. Tissue-engineered models recapitulate many microenvironmental cues present in the human cerebrovasculature (i.e. shear stress) [44]. Similar to results in transwells, we found that the permeability of Lucifer yellow in HD180 microvessels was identical to the value in HD-corrected microvessels $(p=0.691)$ (Fig. 5B, C). However, Lucifer yellow permeability was $\sim 10$-fold lower in 3D microvessel compared to transwell measurements, as also noted previously using a different iPSC source [14]. To assess endothelial cell dynamics in BBB microvessels with HD180 or HD-corrected iBMECs, rates of proliferation (i.e. mitosis) and cell loss (i.e. apoptosis) were tracked from time lapse phase contrast imaging acquired during permeability measurements (Fig. 5D). HD180 microvessels showed $\sim 2$-fold lower rates of proliferation $(p=0.042)$ and cell loss $(p=0.026)$ compared to microvessels formed from HD-corrected cells. Overall turnover rates (proliferation minus cell loss) were similarly lower however had increased variability $(p=0.093)$. These results suggest that HD180 microvessels display unique dynamics of endothelial turnover. Additionally, based on findings of 
altered innate immune responses from GSEA, we determined the adhesion of monocyte-like cells (THP1s) in microvessels. We found that HD180 microvessels displayed elevated adhesion of immune cells compared to microvessels formed from HD-corrected cells $(p=0.033)$ (Fig. 5E). There was an $\sim 3$-fold increase in adhesion despite lack of external inflammatory stimuli (i.e. tumor necrosis factor-a), suggesting that HD180 iBMECs display activated innate immune response.

\section{Discussion}

\section{Summary of changes in BBB phenotype}

Comparison of HD180 to HD-corrected iBMECs (using an optimized differentiation protocol) revealed the following key results: (1) a reduction in TEER but no difference in permeability, (2) a reduction in efflux activity, (3) upregulation of cellular processes associated with HD disease progression, (4) increased cell loss and proliferation, (5) elevated responsiveness to oxidative and osmotic stress, (6) increased responsiveness to VEGF (and elevated expression of VEGFR2), and (7) an increase in immune cell adhesion.. These results imply that paracellular barrier function is maintained in juvenile HD, in addition to a decrease in efflux activity, and a broad increase in vulnerability to pathological perturbations. Additionally, CAG length may modulate the severity of changes in iBMEC phenotype, matching findings that CAG repeat length is associated with the age of HD onset [45].

\section{Differentiation}

Although differentiation of HD-corrected iPSCs was similar to other iPSCs from healthy individuals (e.g. formation of neural tracts), the differentiation of the HD180 iPSCs was substantially different. We have observed slight differences in differentiation trajectory across other iPSCs carrying mutations associated with different neurodegenerative diseases (data not shown) [6]. These differences highlight a key challenge in comparing the function of iBMEC monolayers: how to optimize the conditions for comparison. Since the yield of adherent BMEC-like cells is dependent on seeding density, we performed differentiations over a wide range of experimental variables to identify conditions where iBMECs purity and barrier function were optimized. Such analysis of the differentiation protocol is key to reliable assessment of differences in barrier phenotype due to genetic mutations.

\section{Solute permeability}

While TEER values for HD180 iBMECs were initially lower than for HD-corrected cells, values for both cell types were above $500 \Omega \mathrm{cm}^{2}$ over 10 days. Previous studies have shown that measurable changes in permeability of small molecular weight compounds are observed when the TEER of iBMECs falls below about $500 \Omega \mathrm{cm}^{2}$ [46]. Consistent with this observation, there were no statistically significant differences in permeability for Lucifer yellow or $10 \mathrm{kDa}$ dextran. Although staining of occludin and ZO-1 at cell-cell junctions was reduced in HD180 iBMECs, and expression of genes associated with junctional proteins was also reduced, these differences had no apparent effect on barrier function. Instead, the HD BBB may also be vulnerable to HD-associated stressors including angiogenic, oxidative, and osmotic compounds.

\section{Beyond barrier function}


To explore pathological phenotypes beyond permeability, we exposed iBMECs to hydrogen peroxide, VEGF, and mannitol; our results suggest elevated vulnerability to oxidative, angiogenic, and osmotic stress, which may predispose the BBB to damage during HD progression and highlight potential therapeutic targets. BMECs are sensitive to oxidative cues, which may contribute to BBB dysfunction across many neurological conditions [32]. Interestingly, antioxidants have been explored for treatment of HD [47], and our results suggest that antioxidants could improve disease outcomes based on BBB protection. Additionally, antisense oligonucleotides (ASOs) that target HTT mRNA have been explored for HD treatment via intrathecal injection. Although ASO clinical trials have been recently halted [48], BBBO could be used to improve the distribution of HTT-targeting therapeutics in the human brain.

Comparison to iBMECs differentiated from adult HD iPSCs and to BMECs from adult postmortem HD tissue

We confirm previous reports of GLUT1 ${ }^{+}$and $\mathrm{CD} 31^{+}$cells emerging from differentiation of iPSCs harboring expanded CAG repeats. However, our results contrast those of previous reports using adult HD iBMECs [5]. Previous work utilized a panel of iPSCs with CAG lengths of 28, 33, 60, 66, 71 and 109, which produced TEER values of $\sim 4,250, \sim 4,750, \sim 3,500, \sim 2,750, \sim 100, \sim 200 \Omega \mathrm{cm}^{2}$, respectively. Thus, above 70 CAG repeats TEER values were very low. In contrast, we found that average TEER values for juvenile HD iBMECs regardless of CAG repeat length were above $\sim 1,000 \Omega \mathrm{cm}^{2}$. Our work suggests that changes in barrier function are more nuanced, with $180 \mathrm{CAG}$ repeats still producing cells with high TEER compared to immortalized and primary BMEC cell sources [49]. There are two possible reasons for the observed differences between juvenile HD iBMECs and adult HD iBMECs. First, adult HD iPSCs likely harbor ageinduced epigenetic changes which could result in unique modes of BBB dysfunction [50]. Second, further optimization of the protocol for differentiation of adult HD iBMECs could result in different barrier phenotypes. As described above, the differentiation of juvenile HD-iBMECs was optimized for production of neural tracts and endothelial purity, resulting in relatively high TEER values. Findings on changes in tight junction expression are also different; here we found lower levels tight junction protein expression, while studies of adult HD iBMECs found no substantial difference in occludin and claudin 5 expression using qPCR and western blot [5].

Recent work has characterized differences in gene expression of cerebrovascular cell types between healthy individuals and individuals with HD using single nuclei RNA-sequencing of post-mortem tissue [31]. Only $8 \%$ of genes upregulated in HD180-iBMECs compared to HD-corrected iBMECs are shared with this study, further suggesting that distinct changes in BMECs may occur at early disease states and with the extremely long CAG repeat lengths characteristic of juvenile HD. However, one major similarity between the two studies is evidence of activated immune response. We observed elevated adhesion of immune cells in HD180 iBMEC microvessels despite the lack of external inflammatory stimuli, whereas key initiators and mediators of innate immune activation were upregulated in brain endothelial cells from HD postmortem tissue [31].

\section{Conclusions}


In summary, we showed the impact of expanded CAG repeats on iBMEC phenotype using isogenic juvenile HD iPSCs. CAG expansion in juvenile HD180 iBMECs resulted in lower transendothelial electrical resistance, reduced expression of tight junction proteins, and unique gene expression profiles, but no significant changes in paracellular permeability. However, juvenile HD180 iBMECs displayed increased vulnerability to pathological and therapeutic perturbations including angiogenic factors, oxidative stress, and osmotic stress. We demonstrated that tissue-engineered in vitro BBB models support mechanistic and therapeutic studies of neurodegenerative diseases by exploring unique dynamics of cell turnover and immune cell adhesion. Our results suggest that distinct cerebrovascular changes may occur during juvenile HD that are dependent on the degree of CAG expansion, which should be further explored using isogenic panels that encompass a wider range of CAG repeat lengths [51].

\section{Declarations}

\section{Acknowledgements}

This work was supported by DTRA (HDTRA1-15-1-0046) and NIH (NINDS R01NS106008 and NHLBI R61HL154252). RML acknowledges a National Science Foundation Graduate Research Fellowship under Grant No. DGE1746891. RFN and GG acknowledge a Provost's Undergraduate Research Award (PURA) from the Hopkins Office for Undergraduate Research (HOUR). The authors gratefully acknowledge the Pouladi Lab at the National University of Singapore for providing the isogenic juvenile HD iPSCs.

\section{Author contributions}

RML and PCS conceived the study and wrote the paper. RML, RFN, and PCS designed the in vitro experiments. RML, RFN, DA, GG performed and analyzed 2D experiments. RML and ZG performed and analyzed 3D experiments. All authors reviewed and approved the manuscript.

\section{Conflict of interest}

The authors declare no conflict of interest.

\section{Data availability statement}

The raw/processed data required to reproduce these findings are available from the corresponding author on reasonable request.

\section{References}


1. Bates GP, Dorsey R, Gusella JF, Hayden MR, Kay C, Leavitt BR, Nance M, Ross CA, Scahill RI, Wetzel R, Wild EJ, Tabrizi SJ. Huntington disease. Nat Rev Dis Primers. 2015;1:15005.

2. Zuccato C, Valenza M, Cattaneo E. Molecular mechanisms and potential therapeutical targets in Huntington's disease. Physiol Rev. 2010;90(3):905-81.

3. Drouin-Ouellet J, Sawiak SJ, Cisbani G, Lagace M, Kuan WL, Saint-Pierre M, Dury RJ, Alata W, StAmour I, Mason SL, Calon F, Lacroix S, Gowland PA, Francis ST, Barker RA, Cicchetti F.

Cerebrovascular and blood-brain barrier impairments in Huntington's disease: Potential implications for its pathophysiology, Ann Neurol 78(2) (2015) 160 - 77.

4. Di Pardo A, Amico E, Scalabri F, Pepe G, Castaldo S, Elifani F, Capocci L, De Sanctis C, Comerci L, Pompeo F, D'Esposito M, Filosa S, Crispi S, Maglione V. Impairment of blood-brain barrier is an early event in R6/2 mouse model of Huntington Disease. Sci Rep. 2017;7:41316.

5. Lim RG, Quan C, Reyes-Ortiz AM, Lutz SE, Kedaigle AJ, Gipson TA, Wu J, Vatine GD, Stocksdale J, Casale MS, Svendsen CN, Fraenkel E, Housman DE, Agalliu D, Thompson LM. Huntington's Disease iPSC-Derived Brain Microvascular Endothelial Cells Reveal WNT-Mediated Angiogenic and BloodBrain Barrier Deficits. Cell Rep. 2017;19(7):1365-77.

6. Katt ME, Mayo LN, Ellis SE, Mahairaki V, Rothstein JD, Cheng L, Searson PC. The role of mutations associated with familial neurodegenerative disorders on blood-brain barrier function in an iPSC model. Fluids Barriers CNS. 2019;16(1):20.

7. Lin CY, Hsu YH, Lin MH, Yang TH, Chen HM, Chen YC, Hsiao HY, Chen CC, Chern Y, Chang C. Neurovascular abnormalities in humans and mice with Huntington's disease. Exp Neurol. 2013;250:20-30.

8. Hsiao HY, Chen YC, Huang CH, Chen CC, Hsu YH, Chen HM, Chiu FL, Kuo HC, Chang C, Chern Y. Aberrant astrocytes impair vascular reactivity in Huntington disease. Ann Neurol. 2015;78(2):17892.

9. Chen JJ, Salat DH, Rosas HD. Complex relationships between cerebral blood flow and brain atrophy in early Huntington's disease. Neuroimage. 2012;59(2):1043-51.

10. Cepeda-Prado E, Popp S, Khan U, Stefanov D, Rodriguez J, Menalled LB, Dow-Edwards D, Small SA, Moreno H. R6/2 Huntington's disease mice develop early and progressive abnormal brain metabolism and seizures. J Neurosci. 2012;32(19):6456-67.

11. Barnat M, Capizzi M, Aparicio E, Boluda S, Wennagel D, Kacher R, Kassem R, Lenoir S, Agasse F, Braz BY, Liu JP, Ighil J, Tessier A, Zeitlin SO, Duyckaerts C, Dommergues M, Durr A, Humbert S. Huntington's disease alters human neurodevelopment. Science. 2020;369(6505):787-93.

12. Xu X, Tay Y, Sim B, Yoon SI, Huang Y, Ooi J, Utami KH, Ziaei A, Ng B, Radulescu C, Low D, Ng AYJ, Loh M, Venkatesh B, Ginhoux F, Augustine GJ, Pouladi MA. Reversal of Phenotypic Abnormalities by CRISPR/Cas9-Mediated Gene Correction in Huntington Disease Patient-Derived Induced Pluripotent Stem Cells. Stem Cell Reports. 2017;8(3):619-33.

13. Katt ME, Xu ZS, Gerecht S, Searson PC. Human Brain Microvascular Endothelial Cells Derived from the BC1 iPS Cell Line Exhibit a Blood-Brain Barrier Phenotype. PLoS One. 2016;11(4):e0152105. 
14. Linville RM, DeStefano JG, Nerenberg RF, Grifno GN, Ye R, Gallagher E, Searson PC. Long-term cryopreservation preserves blood-brain barrier phenotype of iPSC-derived brain microvascular endothelial cells and three-dimensional microvessels, Mol Pharm (2020).

15. Neal EH, Marinelli NA, Shi Y, McClatchey PM, Balotin KM, Gullett DR, Hagerla KA, Bowman AB, Ess KC, Wikswo JP, Lippmann ES. A Simplified, Fully Defined Differentiation Scheme for Producing Blood-Brain Barrier Endothelial Cells from Human iPSCs. Stem Cell Reports. 2019;12(6):1380-8.

16. Liao Y, Smyth GK, Shi W. The R package Rsubread is easier, faster, cheaper and better for alignment and quantification of RNA sequencing reads. Nucleic Acids Res. 2019;47(8):e47.

17. Love MI, Huber W, Anders S. Moderated estimation of fold change and dispersion for RNA-seq data with DESeq2. Genome Biol. 2014;15(12):550.

18. Chen EY, Tan CM, Kou Y, Duan Q, Wang Z, Meirelles GV, Clark NR. A. Ma'ayan, Enrichr: interactive and collaborative HTML5 gene list enrichment analysis tool. BMC Bioinformatics. 2013;14:128.

19. Summerfield SG, Read K, Begley DJ, Obradovic T, Hidalgo IJ, Coggon S, Lewis AV, Porter RA, Jeffrey P. Central nervous system drug disposition: the relationship between in situ brain permeability and brain free fraction. J Pharmacol Exp Ther. 2007;322(1):205-13.

20. Linville RM, Arevalo D, Maressa JC, Zhao N, Searson PC. Three-dimensional induced pluripotent stem-cell models of human brain angiogenesis. Microvasc Res. 2020;132:104042.

21. Linville RM, DeStefano JG, Sklar MB, Xu Z, Farrell AM, Bogorad MI, Chu C, Walczak P, Cheng L, Mahairaki V, Whartenby KA, Calabresi PA, Searson PC. Human iPSC-derived blood-brain barrier microvessels: validation of barrier function and endothelial cell behavior, Biomaterials 190-191 (2019) 24-37.

22. Grifno GN, Farrell AM, Linville RM, Arevalo D, Kim JH, Gu L, Searson PC. Tissue-engineered bloodbrain barrier models via directed differentiation of human induced pluripotent stem cells. Sci Rep. 2019;9(1):13957.

23. Chanput W, Mes JJ, Wichers HJ. THP-1 cell line: an in vitro cell model for immune modulation approach. Int Immunopharmacol. 2014;23(1):37-45.

24. Lippmann ES, Al-Ahmad A, Azarin SM, Palecek SP, Shusta EV. A retinoic acid-enhanced, multicellular human blood-brain barrier model derived from stem cell sources. Sci Rep. 2014;4:4160.

25. Stebbins MJ, Lippmann ES, Faubion MG, Daneman R, Palecek SP, Shusta EV. Activation of RARalpha, RARgamma, or RXRalpha Increases Barrier Tightness in Human Induced Pluripotent Stem Cell-Derived Brain Endothelial Cells, Biotechnol J 13(2) (2018).

26. Patel R, Alahmad AJ. Growth-factor reduced Matrigel source influences stem cell derived brain microvascular endothelial cell barrier properties. Fluids Barriers CNS. 2016;13:6.

27. Wilson HK, Canfield SG, Hjortness MK, Palecek SP, Shusta EV. Exploring the effects of cell seeding density on the differentiation of human pluripotent stem cells to brain microvascular endothelial cells. Fluids Barriers CNS. 2015;12:13.

28. The Huntington's Disease Collaborative Research Group. A novel gene containing a trinucleotide repeat that is expanded and unstable on Huntington's disease chromosomes. Cell. 1993;72(6):971- 
83.

29. Langfelder P, Cantle JP, Chatzopoulou D, Wang N, Gao F, Al-Ramahi I, Lu XH, Ramos EM, El-Zein K, Zhao Y, Deverasetty S, Tebbe A, Schaab C, Lavery DJ, Howland D, Kwak S, Botas J, Aaronson JS, Rosinski J, Coppola G, Horvath S, Yang XW. Integrated genomics and proteomics define huntingtin CAG length-dependent networks in mice, Nat Neurosci 19(4) (2016) 623 - 33.

30. Lee H, Fenster RJ, Pineda SS, Gibbs WS, Mohammadi S, Davila-Velderrain J, Garcia FJ, Therrien M, Novis HS, Gao F, Wilkinson H, Vogt T, Kellis M, LaVoie MJ. M. Heiman, Cell Type-Specific Transcriptomics Reveals that Mutant Huntingtin Leads to Mitochondrial RNA Release and Neuronal Innate Immune Activation. Neuron. 2020;107(5):891-908 e8.

31. Garcia FJ, Sun N, Lee H, Godlewski B, Galani K, Mantero J, Bennett DA, Sahin M, Kellis M, Heiman M, Single-cell dissection of the human cerebrovasculature in health and disease, bioRxiv (2021) 2021.04.26.440975.

32. van Leeuwen E, Hampton MB, Smyth LCD. Redox signalling and regulation of the blood-brain barrier. Int J Biochem Cell Biol. 2020;125:105794.

33. Klepac N, Relja M, Klepac R, Hecimovic S, Babic T, Trkulja V. Oxidative stress parameters in plasma of Huntington's disease patients, asymptomatic Huntington's disease gene carriers and healthy subjects: a cross-sectional study. J Neurol. 2007;254(12):1676-83.

34. Chen CM, Wu YR, Cheng ML, Liu JL, Lee YM, Lee PW, Soong BW, Chiu DT. Increased oxidative damage and mitochondrial abnormalities in the peripheral blood of Huntington's disease patients. Biochem Biophys Res Commun. 2007;359(2):335-40.

35. Tang Q, Liu H, Shi XJ, Cheng Y. Blood Oxidative Stress Marker Aberrations in Patients with Huntington's Disease: A Meta-Analysis Study. Oxid Med Cell Longev. 2020;2020:9187195.

36. O'Regan GC, Farag SH, Casey CS, Wood-Kaczmar A, Pocock JM, Tabrizi SJ, Andre R. Human Huntington's disease pluripotent stem cell-derived microglia develop normally but are abnormally hyper-reactive and release elevated levels of reactive oxygen species. J Neuroinflammation. 2021;18(1):94.

37. Machiela E, Jeloka R, Caron NS, Mehta S, Schmidt ME, Baddeley HJE, Tom CM, Polturi N, Xie Y, Mattis VB, Hayden MR, Southwell AL. The Interaction of Aging and Cellular Stress Contributes to Pathogenesis in Mouse and Human Huntington Disease Neurons. Front Aging Neurosci. 2020;12:524369.

38. Kumar A, Ratan RR. Oxidative Stress and Huntington's Disease: The Good, The Bad, and The Ugly. J Huntingtons Dis. 2016;5(3):217-37.

39. Anasooya Shaji C, Robinson BD, Yeager A, Beeram MR, Davis ML, Isbell CL, Huang JH, Tharakan B. The Tri-phasic Role of Hydrogen Peroxide in Blood-Brain Barrier Endothelial cells. Sci Rep. 2019;9(1):133.

40. Munn S, Allanou R, Aschberger K, Berthault F, De Bruijn J, Musset C, O' Connor S, Pakalin S, Pellegrini G, Scheer S, Vegro S. European Union Risk Assessment Report. Hydrogen Peroxide., 2003. 
41. Rapoport SI. Osmotic opening of the blood-brain barrier: principles, mechanism, and therapeutic applications. Cell Mol Neurobiol. 2000;20(2):217-30.

42. Linville RM, DeStefano JG, Sklar MB, Chu C, Walczak P, Searson PC. Modeling hyperosmotic bloodbrain barrier opening within human tissue-engineered in vitro brain microvessels. J Cereb Blood Flow Metab. 2020;40(7):1517-32.

43. Oikari LE, Pandit R, Stewart R, Cuni-Lopez C, Quek H, Sutharsan R, Rantanen LM, Oksanen M, Lehtonen S, de Boer CM, Polo JM, Gotz J, Koistinaho J, White AR. Altered Brain Endothelial Cell Phenotype from a Familial Alzheimer Mutation and Its Potential Implications for Amyloid Clearance and Drug Delivery. Stem Cell Reports. 2020;14(5):924-39.

44. DeStefano JG, Jamieson JJ, Linville RM, Searson PC. Benchmarking in vitro tissue-engineered bloodbrain barrier models. Fluids Barriers CNS. 2018;15(1):32.

45. Genetic Modifiers of Huntington's Disease Consortium. CAG Repeat Not Polyglutamine Length Determines Timing of Huntington's Disease Onset. Cell. 2019;178(4):887-900 e14.

46. Mantle JL, Min L, Lee KH. Minimum Transendothelial Electrical Resistance Thresholds for the Study of Small and Large Molecule Drug Transport in a Human in Vitro Blood-Brain Barrier Model. Mol Pharm. 2016;13(12):4191-8.

47. Johri A, Beal MF. Antioxidants in Huntington's disease. Biochim Biophys Acta. 2012;1822(5):664-74.

48. Wiggins R, Feigin A. Emerging therapeutics in Huntington's disease, Expert Opin Emerg Drugs (2021) 1-8.

49. Helms HC, Abbott NJ, Burek M, Cecchelli R, Couraud PO, Deli MA, Forster C, Galla HJ, Romero IA, Shusta EV, Stebbins MJ, Vandenhaute E, Weksler B, Brodin B. In vitro models of the blood-brain barrier: An overview of commonly used brain endothelial cell culture models and guidelines for their use, J Cereb Blood Flow Metab 36(5) (2016) 862 - 90.

50. Strassler ET, Aalto-Setala K, Kiamehr M, Landmesser U, Krankel N. Age Is Relative-Impact of Donor Age on Induced Pluripotent Stem Cell-Derived Cell Functionality, Front Cardiovasc Med 5 (2018).

51. Ooi J, Langley SR, Xu X, Utami KH, Sim B, Huang Y, Harmston NP, Tay YL, Ziaei A, Zeng R, Low D, Aminkeng F, Sobota RM, Ginhoux F, Petretto E, Pouladi MA. Unbiased Profiling of Isogenic Huntington Disease hPSC-Derived CNS and Peripheral Cells Reveals Strong Cell-Type Specificity of CAG Length Effects. Cell Rep. 2019;26(9):2494-508. e7.

\section{Figures}


A
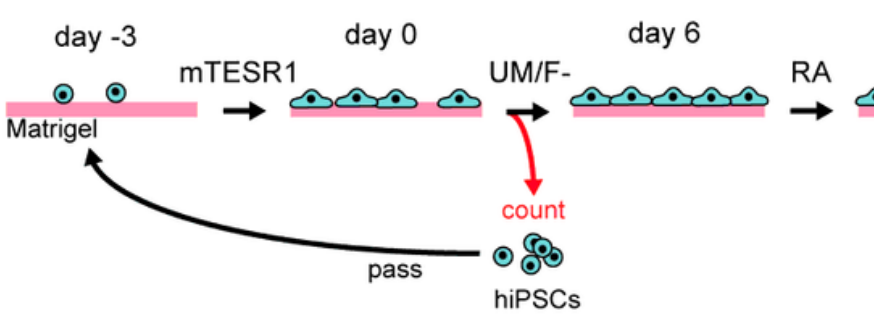

day 8
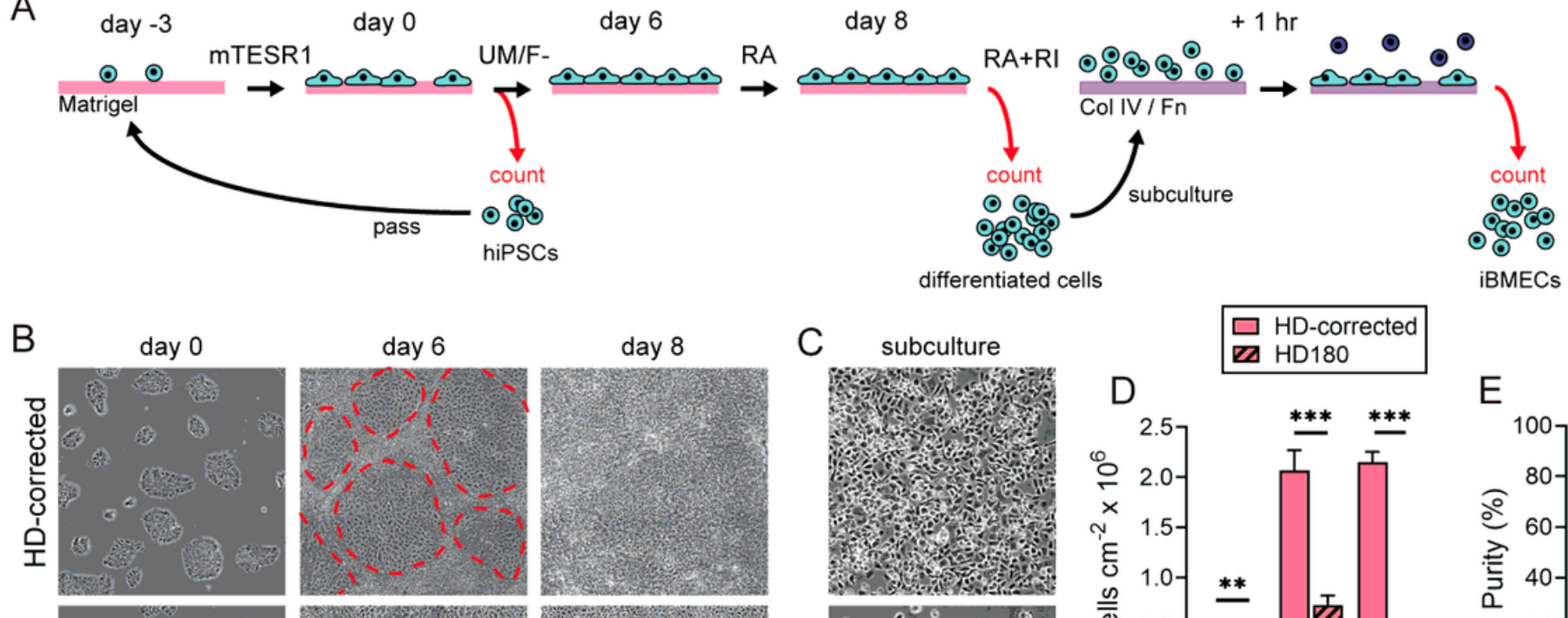

C
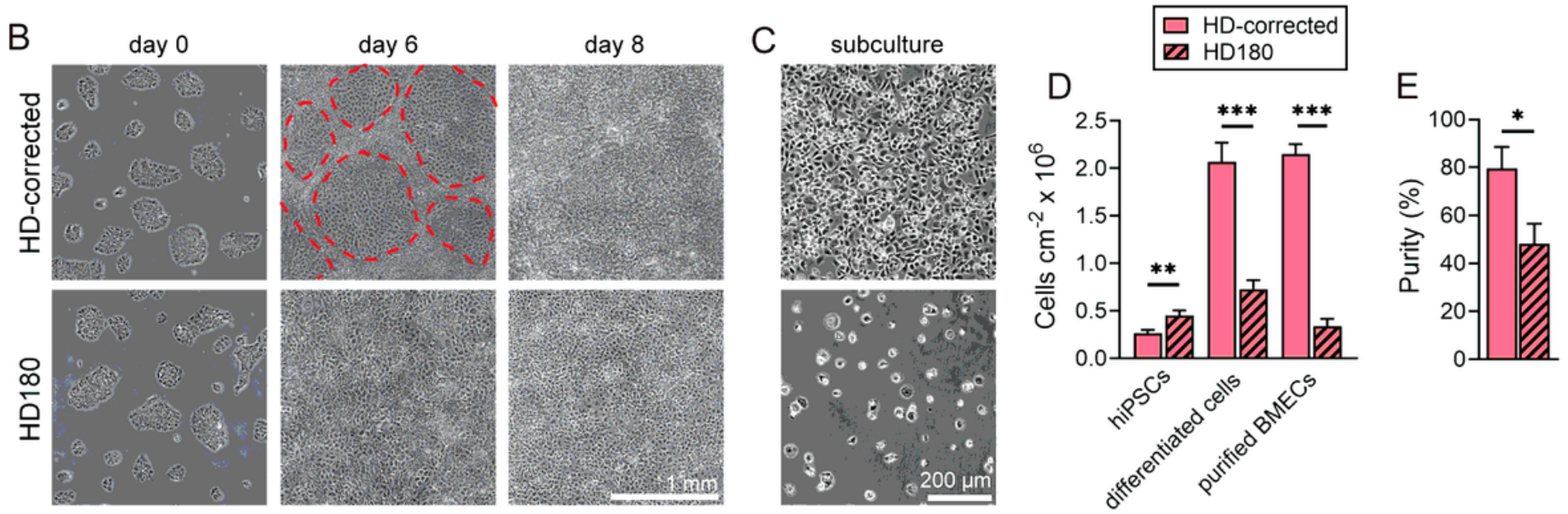

G
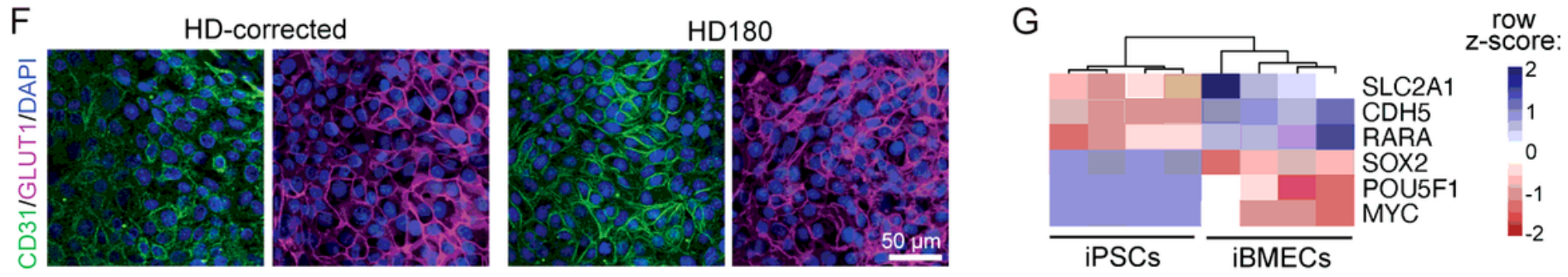

\section{Figure 1}

Comparison of differentiation of HD-corrected and HD180 iPSCs reveals a unique iBMEC differentiation trajectory. (A) Schematic illustration of differentiation timeline. hiPSC colonies are counted and passaged at 10,000 cells $\mathrm{cm}-2$ on Matrigel-coated plates. iBMECs are differentiated over eight days (six-day treatment with UM/F- media and two-day treatment with RA media). (B) Representative phase contrast images of differentiation at day 0, 6, and 8 for HD-corrected and HD180 iPSCs. Endothelial colonies surrounded by neural tracts only form during HD-corrected iBMEC differentiation (red dotted line), despite identical density and appearance of iPSC colonies between the two iPSCs. (C) Representative phase contrast images following subculture of HD-corrected and HD180 differentiated cells highlights the differences in fraction of adherent cells. (D) Cell density on day 0 (hiPSCs three days after passing at 10,000 cm-2), day 8 (differentiated cells), and post-subculture (purified iBMECs). Data collected across $n$ $=7$ and 8 independent differentiations for each cell line, respectively. (E) iBMEC purity (adherent cells: differentiated cells) for HD-corrected and HD180 iBMECs. Data collected across $n=4$ and 5 independent differentiations for each cell line, respectively. (F) Representative immunocytochemical images of CD31 and GLUT-1 for HD-corrected and HD180 iBMECs. (G) iBMEC differentiation downregulates genes associated with pluripotency (POU5F1, SOX2, MYC) independent of CAG repeat length, and upregulates 
genes associated with endothelial and BBB phenotype (CDH5, SLC2A1, RARA). Data represents transformed bulk RNA sequencing data across $n=2$ independent differentiations for HD-corrected and HD180 cells.

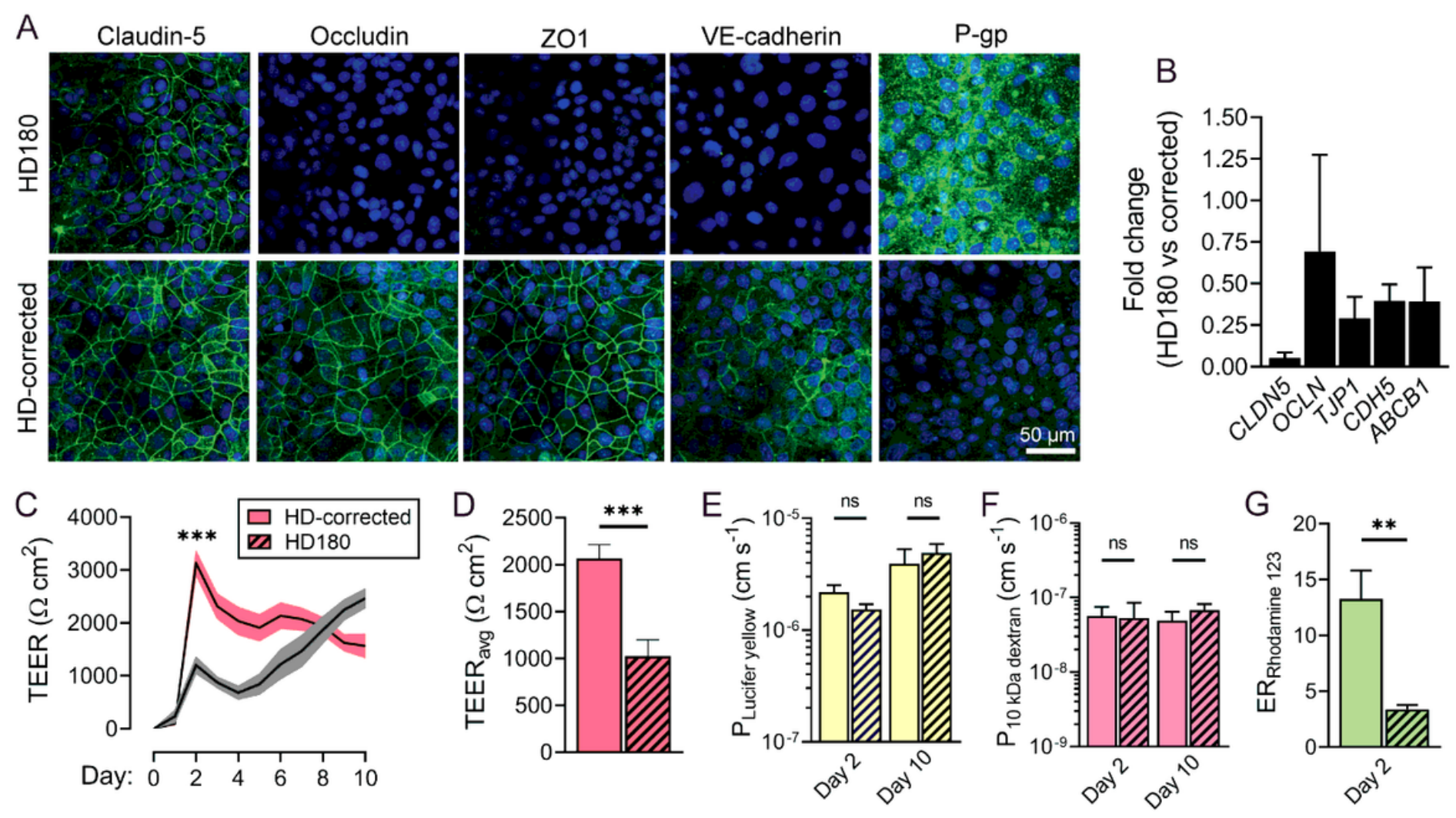

Figure 2

HD180 iBMECs exhibit altered protein localization, gene expression, and BBB function. (A) Immunocytochemistry of BBB proteins (claudin-5, occludin, Z01, P-glycoprotein, GLUT1, CD31, VEcadherin). Representative images are shown. (B) qPCR of BBB proteins: CLDN5, OCLN, TJP1, CDH5, ABCB1. Fold changes compared to HD-corrected iBMECs are shown, with data collected across $n=3$ independent differentiations. (C-D) TEER time course and average over 10 days. Data collected across $n=$ 16 (HD-corrected) 26 (HD180) independent differentiations. (E-F) Lucifer yellow and 10 kDa dextran permeability (day 2 and day 10). Data collected across $n=6$ (HD-corrected) and 8 (HD180) independent differentiations. (G) Rhodamine 123 efflux ratio (day 2). Data collected across $n=5$ independent differentiations for HD-corrected and HD180 iBMECs. (H) Glucose permeability (day 2). Data collected across $n=4$ independent differentiations for HD-corrected and HD180 iBMECs. All recordings in panel C$\mathrm{G}$ represent averages across $\mathrm{n}=2$ - 6 technical replicates (individual transwells) for each biological replicate. 

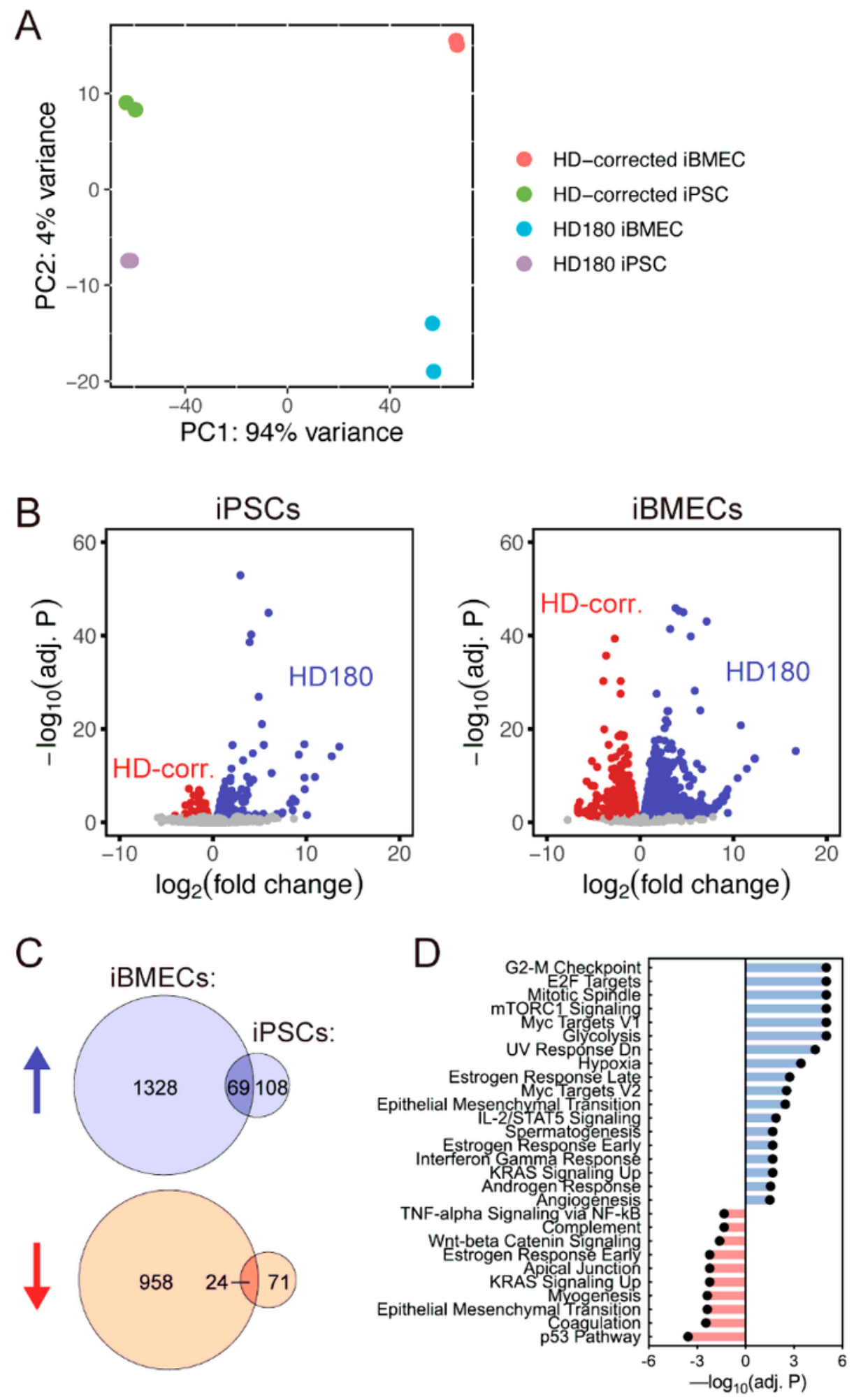

\section{Figure 3}

HD180 iBMECs exhibit unique gene expression profiles. (A) Principle component analysis. Data collected across $n=2$ independent differentiations for each cell line, where collection of iPSC and iBMEC RNA was paired. (B) Volcano plots comparing gene expression of iPSC and iBMECs from HD180 (blue) and HDcorrected (red) sources. (C) Venn diagrams showing overlap of up and downregulated CAG expansiondependent genes in iBMECs versus iPSCs. (D) Hallmark gene sets from the Molecular Signatures 
Database (MSigDB) that are significantly enriched (blue) or depleted (red) in HD180 compared to HDcorrected iBMECs. Significance truncated for gene sets with $-\log 10(\operatorname{adj} . P)>5$.
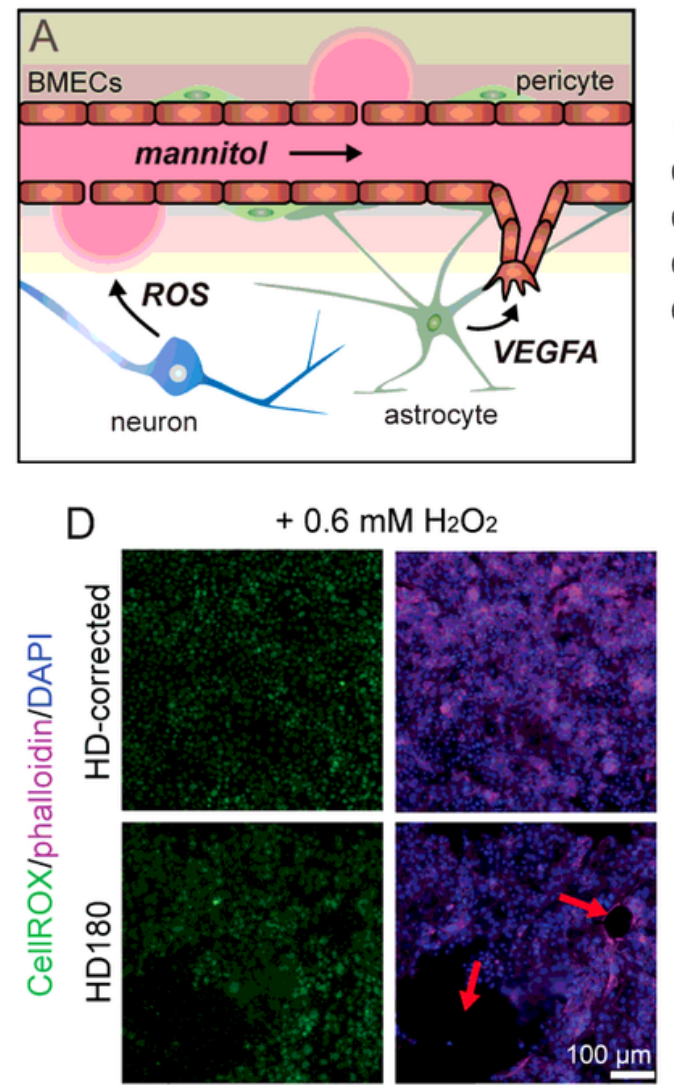

B
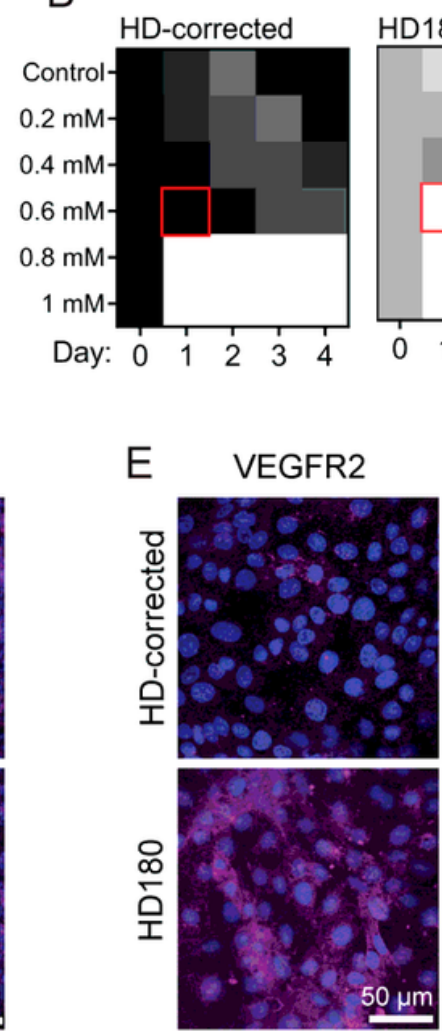

C

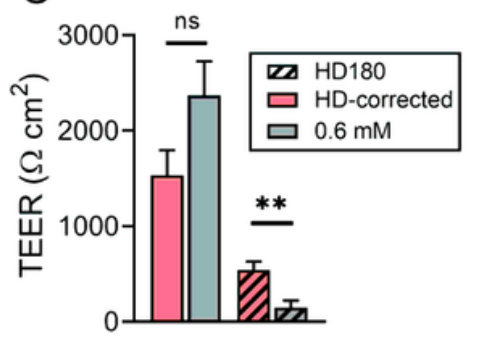

G

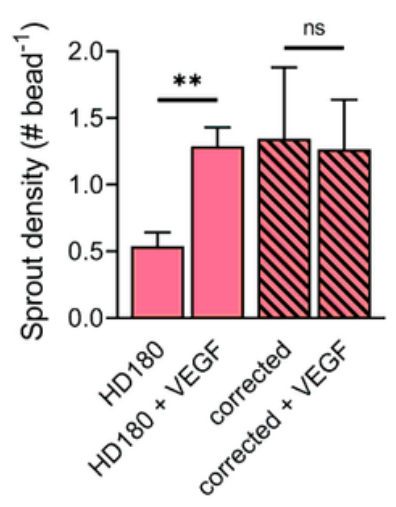

$\mathrm{H}$

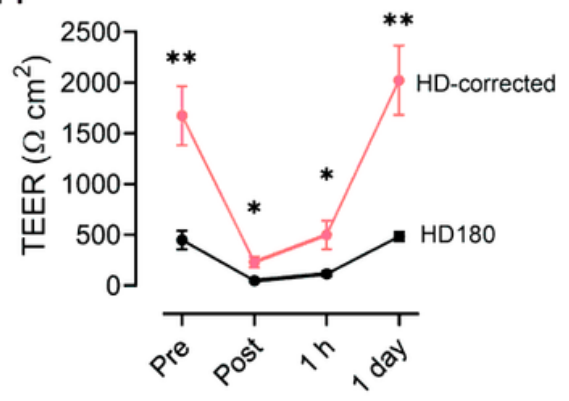

+ VEGF

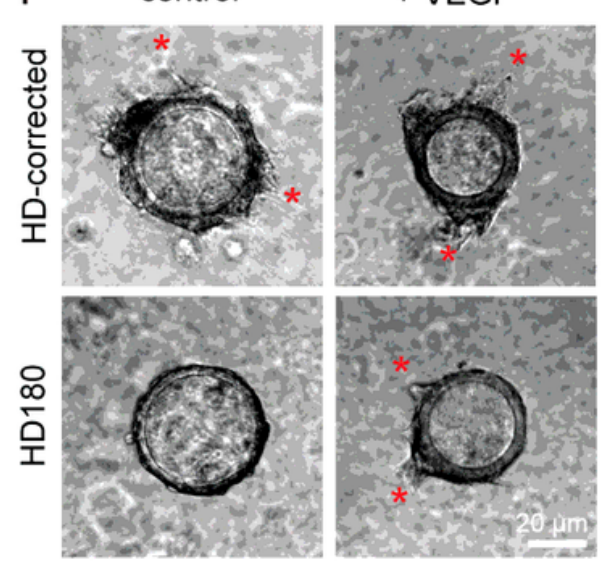

\section{Figure 4}

HD180 iBMECs show increased vulnerability to oxidative, angiogenic, and osmotic stress. (A) Schematic illustration of disease- and therapeutic-relevant perturbations to the HD BBB. (B-D) HD180 iBMECs are more vulnerable to oxidative damage: (B) Time course of iBMEC TEER in response to various $\mathrm{H} 2 \mathrm{O} 2$ concentrations. Red box denotes concentration resulting in most unique responses between iPSCs. (C) iBMEC TEER $24 \mathrm{~h}$ after exposure to $0.6 \mathrm{mM} \mathrm{H2O2}$. Data collected across $\mathrm{n}=7$ (HD180) and 6 (HDcorrected) independent differentiations. (D) Representative fluorescence images of cellular reactive oxygen species, nuclei, and f-actin (Phalloidin) $24 \mathrm{~h}$ after exposure to $0.6 \mathrm{mM}$. Red arrows indicate holes in endothelium. (E) Representative immunocytochemical images of VEGFR2. (F) Bead angiogenesis 
assay. Beads coated in iBMECs seeded in $6 \mathrm{mg} \mathrm{mL}$ collagen I + Matrigel, then supplemented with basal media or with $20 \mathrm{ng} \mathrm{mL-1} \mathrm{bFGF} \mathrm{and} 50 \mathrm{ng}$ mL-1 VEGF. Representative images show beads $72 \mathrm{~h}$ after treatment, where red asterisks denote angiogenic sprouts. (G) Quantification of sprout density across bead angiogenesis assay conditions. Data collected across $n=4$ (HD180) and 3 (HD-corrected) independent differentiations. $(H)$ Changes in TEER in response to osmotic treatment (10 min exposure to 1.4 M mannitol). Data collected across $n=5$ independent differentiations.

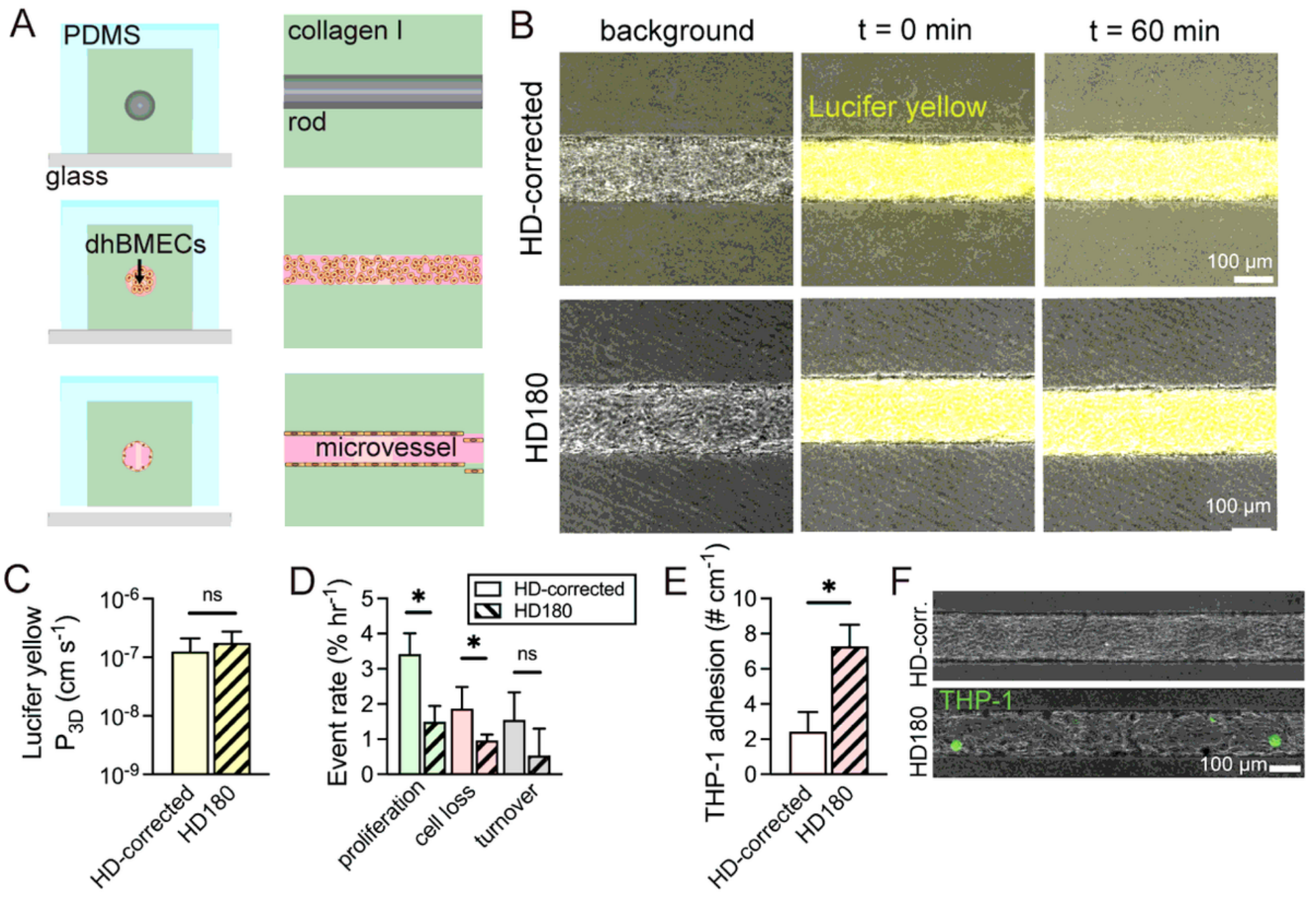

\section{Figure 5}

Tissue-engineered post-capillary venule model of the BBB incorporating HD180 or HD-corrected iBMECs. (A) Schematic illustration of fabrication of three-dimensional microvessels seeded with iBMECs. (B) Microvessels with HD-corrected and HD180 iBMECs similarly restrict Lucifer yellow transport. Representative images are shown. (C) Lucifer yellow permeability in 3D microvessels. Data collected across $n=5$ (HD-corrected) and 4 (HD180 iBMECs) independent differentiations. (D) Turnover rates of microvessels. Data collected across $n=5$ (HD-corrected) and 4 (HD180 iBMECs) independent differentiations. (E-F) Adhesion of monocyte-like cells to tissue-engineered venules: (E) Data collected across $n=4$ (HD-corrected) and 3 (HD180) independent differentiations of iBMECs seeded into microvessels. (F) Representative images of adherent cells after washout. 


\section{Supplementary Files}

This is a list of supplementary files associated with this preprint. Click to download.

- SuppInformationV2.pdf 